\title{
Existence and iterative approximations of nonoscillatory solutions for second order nonlinear neutral delay difference equations
}

Guojing Jiang ${ }^{1}$, Liangshi Zhao ${ }^{2}$ and Shin Min Kang ${ }^{3 *}$

*Correspondence:

smkang@gnu.ac.kr

${ }^{3}$ Department of Mathematics and

RINS, Gyeongsang National University, Jinju, 660-701, Korea

Full list of author information is

available at the end of the article

\section{Abstract}

This paper investigates the second order nonlinear neutral delay difference equation

$$
\begin{aligned}
& \Delta\left[a_{n} \Delta\left(x_{n}+b x_{n-\tau}-d_{n}\right)\right]+\Delta f\left(n, x_{f_{1}(n)}, x_{f_{2}(n)}, \ldots, x_{f_{k}(n)}\right) \\
& +g\left(n, x_{g_{1}(n)}, x_{g_{2}(n)}, \ldots, x_{g_{k}(n)}\right)=c_{n}, \quad n \geq n_{0} .
\end{aligned}
$$

By using the Banach fixed point theorem and some new techniques, we establish the existence results of uncountably many bounded nonoscillatory solutions for the above equation, propose a few Mann type iterative approximation schemes with errors and obtain several errors estimates between the iterative approximations and the nonoscillatory solutions. Examples that cannot be solved by known results are given to illustrate our theorems.

MSC: 39A10; 39A20

Keywords: Second order nonlinear neutral delay difference equation; uncountably many bounded nonoscillatory solutions; Banach fixed point theorem; Mann iterative sequence with errors

\section{Introduction}

In recent years there has been much research activity concerning the oscillation, nonoscillation and existence of solutions for various second order difference equations, for example, see [1-14] and the references therein.

By using the $Z_{p}$ geometrical index theory, Guo and $\mathrm{Yu}$ [4] obtained some sufficient conditions on the multiplicity results of periodic solutions to the second order difference equation

$$
\Delta^{2} x_{n-1}+f\left(x_{n}\right)=0, \quad n \in \mathbb{Z} .
$$

Thandapani et al. [12] gave sufficient conditions for the oscillation of bounded solutions for the second order neutral difference equation

$$
\Delta^{2}\left(x_{n}-p x_{n-k}\right)-q_{n} f\left(x_{n-l}\right)=0, \quad n \geq 1 .
$$

(c) 2015 Jiang et al. This article is distributed under the terms of the Creative Commons Attribution 4.0 International License (http://creativecommons.org/licenses/by/4.0/), which permits unrestricted use, distribution, and reproduction in any medium, provided you give appropriate credit to the original author(s) and the source, provide a link to the Creative Commons license, and indicate if changes were made. 
By applying the contraction principle, Jinfa [5] discussed the existence of a nonoscillatory solution for the second order neutral delay difference equation with positive and negative coefficients

$$
\Delta^{2}\left(x_{n}+p x_{n-m}\right)+p_{n} x_{n-k}-q_{n} x_{n-l}=0, \quad n \geq n_{0}
$$

where $p \in \mathbb{R} \backslash\{-1\}$. Thandapani et al. [13] studied the asymptotic behavior of solutions of the second order neutral difference equations of the form

$$
\Delta^{2}\left(x_{n}+p x_{n-k}\right)+f\left(n, x_{n-l}\right)=0, \quad n \geq 1,
$$

and

$$
\Delta^{2}\left(x_{n}+p x_{n-k}\right)+f\left(n, x_{n-l}, \Delta x_{n-l}\right)=0, \quad n \geq 1,
$$

in terms of some difference inequalities. González and Jiménez-Melado [3] used a fixedpoint theorem derived from the theory of measures of noncompactness to investigate the existence of solutions for the second order difference equation

$$
\Delta\left(q_{n} \Delta x_{n}\right)+f_{n}\left(x_{n}\right)=0, \quad n \geq 0 .
$$

Ma and Guo [9] proved the existence of a nontrivial homoclinic solution for the second order difference equations

$$
\Delta\left(p_{n} \Delta u_{n-1}\right)+q_{n} u_{n}=f\left(n, u_{n}\right), \quad n \in \mathbb{Z}
$$

in terms of the Mountain Pass theorem relying on Ekeland's variational principle and the diagonal method. Yu et al. [14] established the existence of a periodic solution for equation (1.7) by means of the critical point theory. Utilizing the contraction principle, Liu et al. [8] investigated the global existence of solutions for the second order nonlinear neutral delay difference equation

$$
\Delta\left[a_{n} \Delta\left(x_{n}+b x_{n-\tau}\right)\right]+f\left(n, x_{n-d_{1 n}}, x_{n-d_{2 n}}, \ldots, x_{n-d_{k n}}\right)=c_{n}, \quad n \geq n_{0},
$$

relative to all $b \in \mathbb{R}$.

Inspired and motivated by the work in [1-14], we introduce and study the following more general second order nonlinear neutral difference equation:

$$
\begin{aligned}
& \Delta\left[a_{n} \Delta\left(x_{n}+b x_{n-\tau}-d_{n}\right)\right]+\Delta f\left(n, x_{f_{1}(n)}, x_{f_{2}(n)}, \ldots, x_{f_{k}(n)}\right) \\
& \quad+g\left(n, x_{g_{1}(n)}, x_{g_{2}(n)}, \ldots, x_{g_{k}(n)}\right)=c_{n}, \quad n \geq n_{0},
\end{aligned}
$$

where $b \in \mathbb{R}, \tau, k \in \mathbb{N}, n_{0} \in \mathbb{N}_{0},\left\{a_{n}\right\}_{n \in \mathbb{N}_{n_{0}}}$ and $\left\{c_{n}\right\}_{n \in \mathbb{N}_{n_{0}}}$ are real sequences with $a_{n} \neq 0$ for $n \in \mathbb{N}_{n_{0}},\left\{d_{n}\right\}_{n \in \mathbb{N}_{n_{0}}}$ is a bounded sequence, $f, g: \mathbb{N}_{n_{0}} \times \mathbb{R}^{k} \rightarrow \mathbb{R}$ and $f_{l}, g_{l}: \mathbb{N}_{n_{0}} \rightarrow \mathbb{Z}$ with

$$
\lim _{n \rightarrow \infty} f_{l}(n)=\lim _{n \rightarrow \infty} g_{l}(n)=+\infty, \quad l \in\{1,2, \ldots, k\}
$$


Using the Banach fixed point theorem, we obtain sufficient conditions of the existence of uncountably many bounded nonoscillatory solutions for equation (1.9) relative to $b \in$ $\mathbb{R} \backslash\{ \pm 1\}$, suggest a few Mann type iterative approximation methods with errors for these bounded nonoscillatory solutions and study error estimates between the approximation sequences and the bounded nonoscillatory solutions. The results obtained in this paper extend and improve the corresponding results in $[5,8]$. Four nontrivial examples are given to demonstrate the effectiveness of our results.

\section{Preliminaries}

Throughout this paper, we assume that $\Delta$ is the forward difference operator defined by $\Delta x_{n}=x_{n+1}-x_{n}, \Delta^{2} x_{n}=\Delta\left(\Delta x_{n}\right), A$ and $B$ are positive constants with $B>A, \mathbb{R}=(-\infty,+\infty)$, $\mathbb{Z}, \mathbb{N}$ and $\mathbb{N}_{0}$ stand for the sets of all integers, positive integers and nonnegative integers, respectively,

$$
\begin{aligned}
& \mathbb{N}_{n_{0}}=\left\{n: n \in \mathbb{N}_{0} \text { with } n \geq n_{0}\right\}, \quad n_{0} \in \mathbb{N}_{0}, \\
& \alpha=\inf \left\{f_{l}(n), g_{l}(n): 1 \leq l \leq k, n \in \mathbb{N}_{n_{0}}\right\}, \\
& \beta=\min \left\{n_{0}-\tau, \alpha\right\}, \quad \mathbb{Z}_{\beta}=\{n: n \in \mathbb{Z} \text { with } n \geq \beta\}, \\
& A_{n}=d_{n}+A>0, \quad B_{n}=d_{n}+B, \quad n \in \mathbb{Z}_{\beta}, \\
& d_{n}=d_{n_{0}}, \quad \beta \leq n \leq n_{0}-1,
\end{aligned}
$$

and $\bar{d}$ and $\underline{d}$ are two constants with

$$
\underline{d} \leq \inf _{n \in \mathbb{Z}_{\beta}} d_{n}, \quad \bar{d} \geq \sup _{n \in \mathbb{Z}_{\beta}} d_{n} .
$$

Let $l_{\beta}^{\infty}$ denote the Banach space of all bounded sequences in $\mathbb{Z}_{\beta}$ with norm

$$
\|x\|=\sup _{n \in \mathbb{Z}_{\beta}}\left|x_{n}\right| \quad \text { for } x=\left\{x_{n}\right\}_{n \in \mathbb{Z}_{\beta}} \in l_{\beta}^{\infty}
$$

and

$$
\Omega\left(\left\{A_{n}\right\}_{n \in \mathbb{Z}_{\beta}},\left\{B_{n}\right\}_{n \in \mathbb{Z}_{\beta}}\right)=\left\{x=\left\{x_{n}\right\}_{n \in \mathbb{Z}_{\beta}} \in l_{\beta}^{\infty}: A_{n} \leq x_{n} \leq B_{n}, \quad n \in \mathbb{Z}_{\beta}\right\} .
$$

It is easy to see that $\Omega\left(\left\{A_{n}\right\}_{n \in \mathbb{Z}_{\beta}},\left\{B_{n}\right\}_{n \in \mathbb{Z}_{\beta}}\right)$ is a bounded closed and convex subset of $l_{\beta}^{\infty}$.

By a solution of equation (1.9), we mean a sequence $\left\{x_{n}\right\}_{n \in Z_{\beta}}$ with a positive integer $T \geq n_{0}+\tau+|\beta|$ such that equation (1.9) is satisfied for all $n \geq T$. As is customary, a solution of equation (1.9) is said to be oscillatory if it is neither eventually positive nor eventually negative. Otherwise, it is said to be nonoscillatory.

Lemma 2.1 ([15]) Let $\left\{\alpha_{n}\right\}_{n \in \mathbb{N}_{0}},\left\{\beta_{n}\right\}_{n \in \mathbb{N}_{0}},\left\{\gamma_{n}\right\}_{n \in \mathbb{N}_{0}}$ and $\left\{t_{n}\right\}_{n \in \mathbb{N}_{0}}$ be four nonnegative sequences satisfying the inequality

$$
\alpha_{n+1} \leq\left(1-t_{n}\right) \alpha_{n}+t_{n} \beta_{n}+\gamma_{n}, \quad n \in \mathbb{N}_{0}
$$

where $\left\{t_{n}\right\}_{n \in \mathbb{N}_{0}} \subset[0,1], \sum_{n=0}^{\infty} t_{n}=+\infty, \lim _{n \rightarrow \infty} \beta_{n}=0$ and $\sum_{n=0}^{\infty} \gamma_{n}<+\infty$. Then $\lim _{n \rightarrow \infty} \alpha_{n}=0$. 


\section{Existence of uncountably many bounded nonoscillatory solutions}

Now we study the existence of uncountably bounded nonoscillatory solutions for equation (1.9) with respect to $b \in \mathbb{R} \backslash\{ \pm 1\}$, suggest a few Mann iterative approximation schemes with errors for these bounded nonoscillatory solutions and discuss the errors estimates between the iterative approximations and the bounded nonoscillatory solutions.

Theorem 3.1 Let $b \in[0,1), A$ and $B$ be two positive constants with $B>A+\frac{b}{1-b}(\bar{d}-\underline{d})$. Assume that there exist four real sequences $\left\{P_{n}\right\}_{n \in \mathbb{N}_{n_{0}}},\left\{Q_{n}\right\}_{n \in \mathbb{N}_{n_{0}}},\left\{R_{n}\right\}_{n \in \mathbb{N}_{n_{0}}}$ and $\left\{W_{n}\right\}_{n \in \mathbb{N}_{n_{0}}}$ satisfying

$$
\begin{aligned}
& \left|f\left(n, u_{1}, u_{2}, \ldots, u_{k}\right)-f\left(n, \bar{u}_{1}, \bar{u}_{2}, \ldots, \bar{u}_{k}\right)\right| \leq P_{n} \max \left\{\left|u_{l}-\bar{u}_{l}\right|: 1 \leq l \leq k\right\}, \\
& \left|g\left(n, u_{1}, u_{2}, \ldots, u_{k}\right)-g\left(n, \bar{u}_{1}, \bar{u}_{2}, \ldots, \bar{u}_{k}\right)\right| \leq R_{n} \max \left\{\left|u_{l}-\bar{u}_{l}\right|: 1 \leq l \leq k\right\}, \\
& \quad n \in \mathbb{N}_{n_{0}}, u_{l}, \bar{u}_{l} \in[\underline{d}+A, \bar{d}+B], 1 \leq l \leq k ; \\
& \left|f\left(n, u_{1}, u_{2}, \ldots, u_{k}\right)\right| \leq Q_{n}, \quad\left|g\left(n, u_{1}, u_{2}, \ldots, u_{k}\right)\right| \leq W_{n}, \\
& \quad n \in \mathbb{N}_{n_{0}}, u_{l} \in[\underline{d}+A, \bar{d}+B], 1 \leq l \leq k ; \\
& \max \left\{\sum_{i=n_{0}}^{\infty} \frac{1}{\left|a_{i}\right|} \max \left\{P_{i}, Q_{i}\right\}, \sum_{i=n_{0}}^{\infty} \sum_{j=i}^{\infty} \frac{1}{\left|a_{i}\right|} \max \left\{R_{j}, W_{j},\left|c_{j}\right|\right\}\right\}<+\infty .
\end{aligned}
$$

Then

(a) for each $L \in(A+b(\bar{d}+B), B+b(\underline{d}+A))$, there exist $\theta \in(0,1)$ and $T \geq n_{0}+\tau+|\beta|$ such that for any $z_{0}=\left\{z_{0, n}\right\}_{n \in \mathbb{Z}_{\beta}} \in \Omega\left(\left\{A_{n}\right\}_{n \in \mathbb{Z}_{\beta}},\left\{B_{n}\right\}_{n \in \mathbb{Z}_{\beta}}\right)$, the Mann iterative sequence with errors $\left\{z_{m}\right\}_{m \in \mathbb{N}_{0}}$, where $z_{m}=\left\{z_{m, n}\right\}_{n \in \mathbb{Z}_{\beta}} \in \Omega\left(\left\{A_{n}\right\}_{n \in \mathbb{Z}_{\beta}},\left\{B_{n}\right\}_{n \in \mathbb{Z}_{\beta}}\right)$ for all $m \in \mathbb{N}_{0}$, generated by the scheme:

$$
z_{m+1, n}=\left\{\begin{array}{c}
\left(1-\alpha_{m}-\beta_{m}\right) z_{m, n}+\alpha_{m}\left\{L+d_{n}-b z_{m, n-\tau}\right. \\
\quad+\sum_{i=n}^{\infty} \frac{1}{a_{i}}\left[f\left(i, z_{m, f_{1}(i)}, z_{m, f_{2}(i)}, \ldots, z_{m, f_{k}(i)}\right)\right. \\
\left.\left.\quad-\sum_{j=i}^{\infty}\left(g\left(j, z_{m, g_{1}(j)}, z_{m, g_{2}(j)}, \ldots, z_{m, g_{k}(j)}\right)-c_{j}\right)\right]\right\} \\
\quad+\beta_{m} \gamma_{m, n}, \quad n \geq T, m \in \mathbb{N}_{0}, \\
\left(1-\alpha_{m}-\beta_{m}\right) z_{m, T}+\alpha_{m}\left\{L+d_{T}-b z_{m, T-\tau}\right. \\
\quad+\sum_{i=T}^{\infty} \frac{1}{a_{i}}\left[f\left(i, z_{m, f_{1}(i)}, z_{m, f_{2}(i)}, \ldots, z_{m, f_{k}(i)}\right)\right. \\
\left.\left.\quad-\sum_{j=i}^{\infty}\left(g\left(j, z_{m, g_{1}(j)}, z_{m, g_{2}(j)}, \ldots, z_{m, g_{k}(j)}\right)-c_{j}\right)\right]\right\} \\
\quad+\beta_{m} \gamma_{m, T}, \quad \beta \leq n<T, m \in \mathbb{N}_{0}
\end{array}\right.
$$

converges to a bounded nonoscillatory solution $x=\left\{x_{n}\right\}_{n \in \mathbb{Z}_{\beta}} \in \Omega\left(\left\{A_{n}\right\}_{n \in \mathbb{Z}_{\beta}},\left\{B_{n}\right\}_{n \in \mathbb{Z}_{\beta}}\right.$ ) of equation (1.9) and has the following errors estimate:

$$
\left\|z_{m+1}-x\right\| \leq\left(1-(1-\theta) \alpha_{m}\right)\left\|z_{m}-x\right\|+2(\bar{d}+B) \beta_{m}, \quad m \in \mathbb{N}_{0},
$$

where $\left\{\gamma_{m}\right\}_{m \in \mathbb{N}_{0}}$ is an arbitrary sequence in $\Omega\left(\left\{A_{n}\right\}_{n \in \mathbb{Z}_{\beta}},\left\{B_{n}\right\}_{n \in \mathbb{Z}_{\beta}}\right)$ with $\gamma_{m}=\left\{\gamma_{m, n}\right\}_{n \in \mathbb{Z}_{\beta}}$ for each $m \in \mathbb{N}_{0},\left\{\alpha_{m}\right\}_{m \in \mathbb{N}_{0}}$ and $\left\{\beta_{m}\right\}_{m \in \mathbb{N}_{0}}$ are any sequences in $[0,1]$ such that

$$
\sum_{m=0}^{\infty} \alpha_{m}=+\infty
$$


and

$$
\begin{aligned}
& \sum_{m=0}^{\infty} \beta_{m}<+\infty \text { or there exists a sequence }\left\{\xi_{m}\right\}_{m \in \mathbb{N}_{0}} \subset[0,+\infty) \\
& \text { satisfying } \beta_{m}=\xi_{m} \alpha_{m}, m \geq 0 \text { and } \lim _{m \rightarrow \infty} \xi_{m}=0
\end{aligned}
$$

(b) equation (1.9) possesses uncountably many bounded nonoscillatory solutions in $\Omega\left(\left\{A_{n}\right\}_{n \in \mathbb{Z}_{\beta}},\left\{B_{n}\right\}_{n \in \mathbb{Z}_{\beta}}\right)$.

Proof Set $L \in(A+b(\bar{d}+B), B+b(\underline{d}+A))$. It follows from (3.3) and $b \in[0,1)$ that there exist $\theta \in(0,1)$ and $T \geq n_{0}+\tau+|\beta|$ satisfying

$$
\theta=b+\sum_{i=T}^{\infty} \frac{1}{\left|a_{i}\right|}\left(P_{i}+\sum_{j=i}^{\infty} R_{j}\right)
$$

and

$$
\sum_{i=T}^{\infty} \frac{1}{\left|a_{i}\right|}\left[Q_{i}+\sum_{j=i}^{\infty}\left(W_{j}+\left|c_{j}\right|\right)\right] \leq \min \{L-A-b(\bar{d}+B), B+b(\underline{d}+A)-L\} .
$$

In order to prove (i), we now define a mapping $S_{L}: \Omega\left(\left\{A_{n}\right\}_{n \in \mathbb{Z}_{\beta}},\left\{B_{n}\right\}_{n \in \mathbb{Z}_{\beta}}\right) \rightarrow l_{\beta}^{\infty}$ by

$$
\left(S_{L} x\right)_{n}=\left\{\begin{array}{l}
L+d_{n}-b x_{n-\tau}+\sum_{i=n}^{\infty} \frac{1}{a_{i}}\left[f\left(i, x_{f_{1}(i)}, x_{f_{2}(i)}, \ldots, x_{f_{k}(i)}\right)\right. \\
\left.\quad-\sum_{j=i}^{\infty}\left(g\left(j, x_{g_{1}(j)}, x_{g_{2}(j)}, \ldots, x_{g_{k}(j)}\right)-c_{j}\right)\right], \quad n \geq T \\
\left(S_{L} x\right)_{T}, \quad \beta \leq n<T
\end{array}\right.
$$

for any $x=\left\{x_{n}\right\}_{n \in \mathbb{Z}_{\beta}} \in \Omega\left(\left\{A_{n}\right\}_{n \in \mathbb{Z}_{\beta}},\left\{B_{n}\right\}_{n \in \mathbb{Z}_{\beta}}\right)$, and show that $S_{L}$ has a fixed point, which is also a bounded nonoscillatory solution of equation (1.9).

Let $x=\left\{x_{n}\right\}_{n \in \mathbb{Z}_{\beta}}, y=\left\{y_{n}\right\}_{n \in \mathbb{Z}_{\beta}} \in \Omega\left(\left\{A_{n}\right\}_{n \in \mathbb{Z}_{\beta}},\left\{B_{n}\right\}_{n \in \mathbb{Z}_{\beta}}\right)$. In view of (3.1), (3.8), and (3.10), we get for any $n \geq T$

$$
\begin{aligned}
& \left|\left(S_{L} x\right)_{n}-\left(S_{L} y\right)_{n}\right| \\
& =\mid b x_{n-\tau}-b y_{n-\tau}+\sum_{i=n}^{\infty} \frac{1}{a_{i}}\left[f\left(i, x_{f_{1}(i)}, x_{f_{2}(i)}, \ldots, x_{f_{k}(i)}\right)-f\left(i, y_{f_{1}(i)}, y_{f_{2}(i)}, \ldots, y_{f_{k}(i)}\right)\right. \\
& \left.\quad-\sum_{j=i}^{\infty}\left(g\left(j, x_{g_{1}(j)}, x_{g_{2}(j)}, \ldots, x_{g_{k}(j)}\right)-g\left(j, y_{g_{1}(j)}, y_{g_{2}(j)}, \ldots, y_{g_{k}(j)}\right)\right)\right] \mid \\
& \leq b\left|x_{n-\tau}-y_{n-\tau}\right|+\sum_{i=n}^{\infty} \frac{1}{\left|a_{i}\right|}\left[P_{i} \max \left\{\left|x_{f_{l}(i)}-y_{f_{l}(i)}\right|: 1 \leq l \leq k\right\}\right. \\
& \left.\quad+\sum_{j=i}^{\infty} R_{j} \max \left\{\left|x_{g_{l}(j)}-y_{g_{l}(j)}\right|: 1 \leq l \leq k\right\}\right] \\
& \leq b\|x-y\|+\sum_{i=n}^{\infty} \frac{1}{\left|a_{i}\right|}\left(P_{i}+\sum_{j=i}^{\infty} R_{j}\right)\|x-y\| \\
& \leq
\end{aligned}
$$


which leads that

$$
\left\|S_{L} x-S_{L} y\right\| \leq \theta\|x-y\|, \quad x, y \in \Omega\left(\left\{A_{n}\right\}_{n \in \mathbb{Z}_{\beta}},\left\{B_{n}\right\}_{n \in \mathbb{Z}_{\beta}}\right) .
$$

By (3.2), (3.9), and (3.10), we infer that for each $n \geq T$

$$
\begin{aligned}
\left(S_{L} x\right)_{n}= & L+d_{n}-b x_{n-\tau}+\sum_{i=n}^{\infty} \frac{1}{a_{i}}\left[f\left(i, x_{f_{1}(i)}, x_{f_{2}(i)}, \ldots, x_{f_{k}(i)}\right)\right. \\
& \left.-\sum_{j=i}^{\infty}\left(g\left(j, x_{g_{1}(j)}, x_{g_{2}(j)}, \ldots, x_{g_{k}(j)}\right)-c_{j}\right)\right] \\
\leq & L+d_{n}-b(\underline{d}+A)+\sum_{i=T}^{\infty} \frac{1}{\left|a_{i}\right|}\left[Q_{i}+\sum_{j=i}^{\infty}\left(W_{j}+\left|c_{j}\right|\right)\right] \\
\leq & L+d_{n}-b(\underline{d}+A)+\min \{L-A-b(\bar{d}+B), B+b(\underline{d}+A)-L\} \\
\leq & B_{n}
\end{aligned}
$$

and

$$
\begin{aligned}
\left(S_{L} x\right)_{n}= & L+d_{n}-b x_{n-\tau}+\sum_{i=n}^{\infty} \frac{1}{a_{i}}\left[f\left(i, x_{f_{1}(i)}, x_{f_{2}(i)}, \ldots, x_{f_{k}(i)}\right)\right. \\
& \left.-\sum_{j=i}^{\infty}\left(g\left(j, x_{g_{1}(j)}, x_{g_{2}(j)}, \ldots, x_{g_{k}(j)}\right)-c_{j}\right)\right] \\
\geq & L+d_{n}-b(\bar{d}+B)-\sum_{i=T}^{\infty} \frac{1}{\left|a_{i}\right|}\left[Q_{i}+\sum_{j=i}^{\infty}\left(W_{j}+\left|c_{j}\right|\right)\right] \\
\geq & L+d_{n}-b(\bar{d}+B)-\min \{L-A-b(\bar{d}+B), B+b(\underline{d}+A)-L\} \\
\geq & A_{n},
\end{aligned}
$$

which yield

$$
S_{L}\left(\Omega\left(\left\{A_{n}\right\}_{n \in \mathbb{Z}_{\beta}},\left\{B_{n}\right\}_{n \in \mathbb{Z}_{\beta}}\right)\right) \subseteq \Omega\left(\left\{A_{n}\right\}_{n \in \mathbb{Z}_{\beta}},\left\{B_{n}\right\}_{n \in \mathbb{Z}_{\beta}}\right) .
$$

Hence (3.11) and (3.12) mean that $S_{L}$ is a contraction mapping in $\Omega\left(\left\{A_{n}\right\}_{n \in \mathbb{Z}_{\beta}},\left\{B_{n}\right\}_{n \in \mathbb{Z}_{\beta}}\right)$ and it has a unique fixed point $x=\left\{x_{n}\right\}_{n \in \mathbb{Z}_{\beta}} \in \Omega\left(\left\{A_{n}\right\}_{n \in \mathbb{Z}_{\beta}},\left\{B_{n}\right\}_{n \in \mathbb{Z}_{\beta}}\right)$. It follows from (3.10) that

$$
\begin{aligned}
x_{n}= & L+d_{n}-b x_{n-\tau}+\sum_{i=n}^{\infty} \frac{1}{a_{i}}\left[f\left(i, x_{f_{1}(i)}, x_{f_{2}(i)}, \ldots, x_{f_{k}(i)}\right)\right. \\
& \left.-\sum_{j=i}^{\infty}\left(g\left(j, x_{g_{1}(j)}, x_{g_{2}(j)}, \ldots, x_{g_{k}(j)}\right)-c_{j}\right)\right], \quad n \geq T,
\end{aligned}
$$


which gives

$$
\begin{aligned}
\Delta\left(x_{n}+b x_{n-\tau}-d_{n}\right)= & -\frac{1}{a_{n}}\left[f\left(n, x_{f_{1}(n)}, x_{f_{2}(n)}, \ldots, x_{f_{k}(n)}\right)\right. \\
& \left.-\sum_{j=n}^{\infty}\left(g\left(j, x_{g_{1}(j)}, x_{g_{2}(j)}, \ldots, x_{g_{k}(j)}\right)-c_{j}\right)\right], \quad n \geq T,
\end{aligned}
$$

and

$$
\begin{aligned}
& \Delta\left(a_{n} \Delta\left(x_{n}+b x_{n-\tau}-d_{n}\right)\right)+\Delta f\left(n, x_{f_{1}(n)}, x_{f_{2}(n)}, \ldots, x_{f_{k}(n)}\right) \\
& \quad+g\left(n, x_{g_{1}(n)}, x_{g_{2}(n)}, \ldots, x_{g_{k}(n)}\right)=c_{n}, \quad n \geq T .
\end{aligned}
$$

That is, the fixed point $x=\left\{x_{n}\right\}_{n \in \mathbb{Z}_{\beta}}$ of $S_{L}$ in $\Omega\left(\left\{A_{n}\right\}_{n \in \mathbb{Z}_{\beta}},\left\{B_{n}\right\}_{n \in \mathbb{Z}_{\beta}}\right)$ is a bounded nonoscillatory solution of equation (1.9).

In light of (3.4), (3.8), (3.10), and (3.11), we deduce that for all $n \geq T$ and $m \in \mathbb{N}_{0}$

$$
\begin{aligned}
\left|z_{m+1, n}-x_{n}\right| & \mid\left(1-\alpha_{m}-\beta_{m}\right) z_{m, n}+\alpha_{m}\left\{L+d_{n}-b z_{m, n-\tau}\right. \\
= & +\sum_{i=n}^{\infty} \frac{1}{a_{i}}\left[f\left(i, z_{m, f_{1}(i)}, z_{m, f_{2}(i)}, \ldots, z_{m, f_{k}(i)}\right)\right. \\
& \left.\left.\quad-\sum_{j=i}^{\infty}\left(g\left(j, z_{m, g_{1}(j)}, z_{m, g_{2}(j)}, \ldots, z_{m, g_{k}(j)}\right)-c_{j}\right)\right]\right\}+\beta_{m} \gamma_{m, n}-x_{n} \mid \\
\leq & \left(1-\alpha_{m}-\beta_{m}\right)\left|z_{m, n}-x_{n}\right|+\alpha_{m}\left|\left(S_{L} z_{m}\right)_{n}-\left(S_{L} x\right)_{n}\right|+\beta_{m}\left|\gamma_{m, n}-x_{n}\right| \\
\leq & \left(1-\alpha_{m}-\beta_{m}\right)\left\|z_{m}-x\right\|+\alpha_{m} \theta\left\|z_{m}-x\right\|+2(\bar{d}+B) \beta_{m} \\
\leq & \left(1-(1-\theta) \alpha_{m}\right)\left\|z_{m}-x\right\|+2(\bar{d}+B) \beta_{m},
\end{aligned}
$$

which implies that (3.5) holds. It follows from (3.6), (3.7), and Lemma 2.1 that $\lim _{m \rightarrow \infty} z_{m}=x$.

Next we prove (ii). It follows from (i) that for any distinct $L_{1}, L_{2} \in(A+b(\bar{d}+B), B+b(\underline{d}+$ $A)$ ), there exist $\theta_{1}, \theta_{2} \in(0,1)$ and $T_{1}, T_{2} \geq n_{0}+\tau+|\beta|$ satisfying (3.8)-(3.10), where $\theta, T, L$ and $S_{L}$ are replaced by $\theta_{j}, T_{j}, L_{j}$ and $S_{T_{j}}, j \in\{1,2\}$, respectively. In view of (3.1) there exists $T_{3}>\max \left\{T_{1}, T_{2}\right\}$ satisfying

$$
\sum_{i=T_{3}}^{\infty} \frac{1}{\left|a_{i}\right|}\left(P_{i}+\sum_{j=i}^{\infty} R_{j}\right)<\frac{\left|L_{1}-L_{2}\right|}{4(\bar{d}+B)} .
$$

Obviously, the contraction mappings $S_{L_{1}}$ and $S_{L_{2}}$ have the unique fixed points $x=$ $\left\{x_{n}\right\}_{n \in \mathbb{Z}_{\beta}}, y=\left\{y_{n}\right\}_{n \in \mathbb{Z}_{\beta}} \in \Omega\left(\left\{A_{n}\right\}_{n \in \mathbb{Z}_{\beta}},\left\{B_{n}\right\}_{n \in \mathbb{Z}_{\beta}}\right)$, respectively. That is, $x$ and $y$ are bounded nonoscillatory solutions of equation (1.9) in $\Omega\left(\left\{A_{n}\right\}_{n \in \mathbb{Z}_{\beta}},\left\{B_{n}\right\}_{n \in \mathbb{Z}_{\beta}}\right)$. In the following, we 
show only that $x \neq y$. In view of (3.10), we arrive at

$$
\begin{aligned}
x_{n}= & L_{1}+d_{n}-b x_{n-\tau}+\sum_{i=n}^{\infty} \frac{1}{a_{i}}\left[f\left(i, x_{f_{1}(i)}, x_{f_{2}(i)}, \ldots, x_{f_{k}(i)}\right)\right. \\
& \left.-\sum_{j=i}^{\infty}\left(g\left(j, x_{g_{1}(j)}, x_{g_{2}(j)}, \ldots, x_{g_{k}(j)}\right)-c_{j}\right)\right], \quad n \geq T_{3}
\end{aligned}
$$

and

$$
\begin{aligned}
y_{n}= & L_{2}+d_{n}-b y_{n-\tau}+\sum_{i=n}^{\infty} \frac{1}{a_{i}}\left[f\left(i, y_{f_{1}(i)}, y_{f_{2}(i)}, \ldots, y_{f_{k}(i)}\right)\right. \\
& \left.-\sum_{j=i}^{\infty}\left(g\left(j, y_{g_{1}(j)}, y_{g_{2}(j)}, \ldots, y_{g_{k}(j)}\right)-c_{j}\right)\right], \quad n \geq T_{3},
\end{aligned}
$$

which together with (3.13) yield

$$
\begin{aligned}
\left|\left(x_{n}-y_{n}\right)+b\left(x_{n-\tau}-y_{n-\tau}\right)\right| & \mid L_{1}-L_{2}+\sum_{i=n}^{\infty} \frac{1}{a_{i}}\left\{f\left(i, x_{f_{1}(i)}, x_{f_{2}(i)}, \ldots, x_{f_{k}(i)}\right)-f\left(i, y_{f_{1}(i)}, y_{f_{2}(i)}, \ldots, y_{f_{k}(i)}\right)\right. \\
& \left.-\sum_{j=i}^{\infty}\left[g\left(j, x_{g_{1}(j)}, x_{g_{2}(j)}, \ldots, x_{g_{k}(j)}\right)-g\left(j, y_{g_{1}(j)}, y_{g_{2}(j)}, \ldots, y_{g_{k}(j)}\right)\right]\right\} \mid \\
\geq & \left|L_{1}-L_{2}\right|-\sum_{i=n}^{\infty} \frac{1}{\left|a_{i}\right|}\left[P_{i} \max \left\{\left|x_{f_{l}(i)}-y_{f_{l}(i)}\right|: 1 \leq l \leq k\right\}\right. \\
& \left.+\sum_{j=i}^{\infty} R_{j} \max \left\{\left|x_{g_{l}(j)}-y_{g_{l}(j)}\right|: 1 \leq l \leq k\right\}\right] \\
\geq & \left|L_{1}-L_{2}\right|-\sum_{i=n}^{\infty} \frac{1}{\left|a_{i}\right|}\left(P_{i}+\sum_{j=i}^{\infty} R_{j}\right)\|x-y\| \\
\geq & 0, \quad\left|L_{1}-L_{2}\right|-2(\bar{d}+B) \sum_{i=T_{3}}^{\infty} \frac{1}{\left|a_{i}\right|}\left(P_{i}+\sum_{j=i}^{+\infty} R_{j}\right) \\
\geq & \frac{\left|L_{1}-L_{2}\right|}{2} \\
& n
\end{aligned}
$$

that is, $x \neq y$. This completes the proof.

Theorem 3.2 Let $b \in(-1,0], A$ and $B$ be two positive constants with $B>A+\frac{b}{1+b}(\underline{d}-\bar{d})$. Assume that there exist four real sequences $\left\{P_{n}\right\}_{n \in \mathbb{N}_{n_{0}}},\left\{Q_{n}\right\}_{n \in \mathbb{N}_{n_{0}}},\left\{R_{n}\right\}_{n \in \mathbb{N}_{n_{0}}}$ and $\left\{W_{n}\right\}_{n \in \mathbb{N}_{n_{0}}}$ satisfying (3.1)-(3.3). Then

(a) for any $L \in(A+b(\underline{d}+A), B+b(\bar{d}+B))$, there exist $\theta \in(0,1)$ and $T \geq n_{0}+\tau+|\beta|$ such that for each $z_{0}=\left\{z_{0, n}\right\}_{n \in \mathbb{Z}_{\beta}} \in \Omega\left(\left\{A_{n}\right\}_{n \in \mathbb{Z}_{\beta}},\left\{B_{n}\right\}_{n \in \mathbb{Z}_{\beta}}\right)$, the Mann iterative sequence with errors $\left\{z_{m}\right\}_{m \in \mathbb{N}_{0}}$ generated by (3.4) with $z_{m}=\left\{z_{m, n}\right\}_{n \in \mathbb{Z}_{\beta}} \in \Omega\left(\left\{A_{n}\right\}_{n \in \mathbb{Z}_{\beta}},\left\{B_{n}\right\}_{n \in \mathbb{Z}_{\beta}}\right.$ for all $m \in$ 
$\mathbb{N}_{0}$ converges to a bounded nonoscillatory solution $x=\left\{x_{n}\right\}_{n \in \mathbb{Z}_{\beta}} \in \Omega\left(\left\{A_{n}\right\}_{n \in \mathbb{Z}_{\beta}},\left\{B_{n}\right\}_{n \in \mathbb{Z}_{\beta}}\right)$ of equation (1.9) and satisfies (3.5), where $\left\{\gamma_{m}\right\}_{m \in \mathbb{N}_{0}}$ is an arbitrary sequence in $\Omega\left(\left\{A_{n}\right\}_{n \in \mathbb{Z}_{\beta}}\right.$, $\left\{B_{n}\right\}_{n \in \mathbb{Z}_{\beta}}$ ) with $\gamma_{m}=\left\{\gamma_{m, n}\right\}_{n \in \mathbb{Z}_{\beta}}$ for each $m \in \mathbb{N}_{0},\left\{\alpha_{m}\right\}_{m \in \mathbb{N}_{0}}$ and $\left\{\beta_{m}\right\}_{m \in \mathbb{N}_{0}}$ are any sequences in $[0,1]$ satisfying (3.6) and (3.7);

(b) equation (1.9) possesses uncountably many bounded nonoscillatory solutions in $\Omega\left(\left\{A_{n}\right\}_{n \in \mathbb{Z}_{\beta}},\left\{B_{n}\right\}_{n \in \mathbb{Z}_{\beta}}\right)$.

Proof Let $L \in(A+b(\underline{d}+A), B+b(\bar{d}+B))$. On account of (3.3) and $b \in(-1,0]$, there exist $\theta \in(0,1)$ and $T \geq n_{0}+\tau+|\beta|$ satisfying

$$
\theta=-b+\sum_{i=T}^{\infty} \frac{1}{\left|a_{i}\right|}\left(P_{i}+\sum_{j=i}^{\infty} R_{j}\right)
$$

and

$$
\sum_{i=T}^{\infty} \frac{1}{\left|a_{i}\right|}\left[Q_{i}+\sum_{j=i}^{\infty}\left(W_{j}+\left|c_{j}\right|\right)\right] \leq \min \{L-A-b(\underline{d}+A), B+b(\bar{d}+B)-L\} .
$$

Let the mapping $S_{L}: \Omega\left(\left\{A_{n}\right\}_{n \in \mathbb{Z}_{\beta}},\left\{B_{n}\right\}_{n \in \mathbb{Z}_{\beta}}\right) \rightarrow l_{\beta}^{\infty}$ be defined by (3.10). The rest of the proof is similar to that of Theorem 3.1 and is omitted. This completes the proof.

Theorem 3.3 Let $b \in(1,+\infty), A$ and $B$ be two positive constants with $B>A+\frac{b+2}{b-1}(\bar{d}-\underline{d})$. Assume that there exist four real sequences $\left\{P_{n}\right\}_{n \in \mathbb{N}_{n_{0}}},\left\{Q_{n}\right\}_{n \in \mathbb{N}_{n_{0}}},\left\{R_{n}\right\}_{n \in \mathbb{N}_{n_{0}}}$ and $\left\{W_{n}\right\}_{n \in \mathbb{N}_{n_{0}}}$ satisfying (3.1)-(3.3). Then

(a) for any $L \in(B+b(\bar{d}+A)+\bar{d}-\underline{d}, A+b(\underline{d}+B)+\underline{d}-\bar{d})$, there exist $\theta \in(0,1)$ and $T \geq$ $n_{0}+\tau+|\beta|$ such that for each $z_{0}=\left\{z_{0, n}\right\}_{n \in \mathbb{Z}_{\beta}} \in \Omega\left(\left\{A_{n}\right\}_{n \in \mathbb{Z}_{\beta}},\left\{B_{n}\right\}_{n \in \mathbb{Z}_{\beta}}\right)$, the Mann iterative sequence with errors $\left\{z_{m}\right\}_{m \in \mathbb{N}_{0}}$, where $z_{m}=\left\{z_{m, n}\right\}_{n \in \mathbb{Z}_{\beta}} \in \Omega\left(\left\{A_{n}\right\}_{n \in \mathbb{Z}_{\beta}},\left\{B_{n}\right\}_{n \in \mathbb{Z}_{\beta}}\right)$ for all $m \in$ $\mathbb{N}_{0}$, generated by the schemes:

$$
z_{m+1, n}=\left\{\begin{aligned}
(1 & \left.-\alpha_{m}-\beta_{m}\right) z_{m, n}+\alpha_{m}\left\{\frac{L}{b}+\frac{d_{n+\tau}}{b}-\frac{z_{m, n+\tau}}{b}\right. \\
& +\frac{1}{b} \sum_{i=n+\tau}^{\infty} \frac{1}{a_{i}}\left[f\left(i, z_{m, f_{1}(i)}, z_{m, f_{2}(i)}, \ldots, z_{m, f_{k}(i)}\right)\right. \\
& \left.\left.-\sum_{j=i}^{\infty}\left(g\left(j, z_{m, g_{1}(j)}, z_{m, g_{2}(j)}, \ldots, z_{m, g_{k}(j)}\right)-c_{j}\right)\right]\right\} \\
& +\beta_{m} \gamma_{m, n}, \quad n \geq T, m \in \mathbb{N}_{0}, \\
(1- & \left.\alpha_{m}-\beta_{m}\right) z_{m, T}+\alpha_{m}\left\{\frac{L}{b}+\frac{d_{T+\tau}}{b}-\frac{z_{m, T+\tau}}{b}\right. \\
& +\frac{1}{b} \sum_{i=T+\tau}^{+\infty} \frac{1}{a_{i}}\left[f\left(i, z_{m, f_{1}(i)}, z_{m, f_{2}(i)}, \ldots, z_{m, f_{k}(i)}\right)\right. \\
& \left.\left.-\sum_{j=i}^{\infty}\left(g\left(j, z_{m, g_{1}(j)}, z_{m, g_{2}(j)}, \ldots, z_{m, g_{k}(j)}\right)-c_{j}\right)\right]\right\} \\
& +\beta_{m} \gamma_{m, T}, \quad \beta \leq n<T, m \in \mathbb{N}_{0},
\end{aligned}\right.
$$

converges to a bounded nonoscillatory solution $x=\left\{x_{n}\right\}_{n \in \mathbb{Z}_{\beta}} \in \Omega\left(\left\{A_{n}\right\}_{n \in \mathbb{Z}_{\beta}},\left\{B_{n}\right\}_{n \in \mathbb{Z}_{\beta}}\right.$ ) of equation (1.9) and satisfies (3.5), where $\left\{\gamma_{m}\right\}_{m \in \mathbb{N}_{0}}$ is an arbitrary sequence in $\Omega\left(\left\{A_{n}\right\}_{n \in \mathbb{Z}_{\beta}}\right.$, $\left.\left\{B_{n}\right\}_{n \in \mathbb{Z}_{\beta}}\right)$ with $\gamma_{m}=\left\{\gamma_{m, n}\right\}_{n \in \mathbb{Z}_{\beta}}$ for each $m \in \mathbb{N}_{0},\left\{\alpha_{m}\right\}_{m \in \mathbb{N}_{0}}$ and $\left\{\beta_{m}\right\}_{m \in \mathbb{N}_{0}}$ are any sequences in $[0,1]$ satisfying (3.6) and (3.7);

(b) equation (1.9) possesses uncountably many bounded nonoscillatory solutions in $\Omega\left(\left\{A_{n}\right\}_{n \in \mathbb{Z}_{\beta}},\left\{B_{n}\right\}_{n \in \mathbb{Z}_{\beta}}\right)$. 
Proof Set $L \in(B+b(\bar{d}+A)+\bar{d}-\underline{d}, A+b(\underline{d}+B)+\underline{d}-\bar{d})$. In view of (3.3) and $b \in(1,+\infty)$, there exist $\theta \in(0,1)$ and $T \geq n_{0}+\tau+|\beta|$ such that

$$
\theta=\frac{1}{b}+\frac{1}{b} \sum_{i=T}^{\infty} \frac{1}{\left|a_{i}\right|}\left(P_{i}+\sum_{j=i}^{\infty} R_{j}\right)
$$

and

$$
\begin{aligned}
& \sum_{i=T}^{\infty} \frac{1}{\left|a_{i}\right|}\left[Q_{i}+\sum_{j=i}^{\infty}\left(W_{j}+\left|c_{j}\right|\right)\right] \\
& \quad \leq \min \{b(\underline{d}+B)+\underline{d}+A-\bar{d}-L, L-b(\bar{d}+A)-\bar{d}-B+\underline{d}\} .
\end{aligned}
$$

Define a mapping $S_{L}: \Omega\left(\left\{A_{n}\right\}_{n \in \mathbb{Z}_{\beta}},\left\{B_{n}\right\}_{n \in \mathbb{Z}_{\beta}}\right) \rightarrow l_{\beta}^{\infty}$ by

$$
\left(S_{L} x\right)_{n}=\left\{\begin{array}{c}
\frac{L}{b}+\frac{d_{n+\tau}}{b}-\frac{x_{n+\tau}}{b}+\frac{1}{b} \sum_{i=n+\tau}^{\infty} \frac{1}{a_{i}}\left[f\left(i, x_{f_{1}(i)}, x_{f_{2}(i)}, \ldots, x_{f_{k}(i)}\right)\right. \\
\left.\quad-\sum_{j=i}^{\infty}\left(g\left(j, x_{g_{1}(j)}, x_{g_{2}(j)}, \ldots, x_{g_{k}(j)}\right)-c_{j}\right)\right], \quad n \geq T \\
\left(S_{L} x\right)_{T}, \quad \beta \leq n<T
\end{array}\right.
$$

for any $x=\left\{x_{n}\right\}_{n \in \mathbb{Z}_{\beta}} \in \Omega\left(\left\{A_{n}\right\}_{n \in \mathbb{Z}_{\beta}},\left\{B_{n}\right\}_{n \in \mathbb{Z}_{\beta}}\right)$.

Let $x=\left\{x_{n}\right\}_{n \in \mathbb{Z}_{\beta}}, y=\left\{y_{n}\right\}_{n \in \mathbb{Z}_{\beta}} \in \Omega\left(\left\{A_{n}\right\}_{n \in \mathbb{Z}_{\beta}},\left\{B_{n}\right\}_{n \in \mathbb{Z}_{\beta}}\right.$ ). Using (3.1), (3.17), and (3.19), we deduce that for any $n \geq T$

$$
\begin{aligned}
\left|\left(S_{L} x\right)_{n}-\left(S_{L} y\right)_{n}\right| \leq & \frac{1}{b}\left|x_{n+\tau}-y_{n+\tau}\right| \\
& +\frac{1}{b} \sum_{i=n+\tau}^{\infty} \frac{1}{\left|a_{i}\right|}\left[\left|f\left(i, x_{f_{1}(i)}, x_{f_{2}(i)}, \ldots, x_{f_{k}(i)}\right)-f\left(i, y_{f_{1}(i)}, y_{f_{2}(i)}, \ldots, y_{f_{k}(i)}\right)\right|\right. \\
& \left.+\sum_{j=i}^{\infty}\left|g\left(j, x_{g_{1}(j)}, x_{g_{2}(j)}, \ldots, x_{g_{k}(j)}\right)-g\left(j, y_{g_{1}(j)}, y_{g_{2}(j)}, \ldots, y_{g_{k}(j)}\right)\right|\right] \\
\leq & \frac{1}{b}\|x-y\|+\frac{1}{b} \sum_{i=n+\tau}^{\infty} \frac{1}{\left|a_{i}\right|}\left(P_{i}+\sum_{j=i}^{\infty} R_{j}\right)\|x-y\| \\
\leq & \theta\|x-y\|,
\end{aligned}
$$

which means (3.11).

By (3.2), (3.18), and (3.19), we infer that for each $n \geq T$

$$
\begin{aligned}
\left(S_{L} x\right)_{n}= & \frac{L}{b}+\frac{d_{n+\tau}}{b}-\frac{x_{n+\tau}}{b}+\frac{1}{b} \sum_{i=n+\tau}^{\infty} \frac{1}{a_{i}}\left[f\left(i, x_{f_{1}(i)}, x_{f_{2}(i)}, \ldots, x_{f_{k}(i)}\right)\right. \\
& \left.-\sum_{j=i}^{\infty}\left(g\left(j, x_{g_{1}(j)}, x_{g_{2}(j)}, \ldots, x_{g_{k}(j)}\right)-c_{j}\right)\right] \\
\leq & \frac{L}{b}+\frac{\bar{d}}{b}-\frac{d}{b}+A \\
b & \frac{1}{b} \sum_{i=n+\tau}^{\infty} \frac{1}{\left|a_{i}\right|}\left[Q_{i}+\sum_{j=i}^{\infty}\left(W_{j}+\left|c_{j}\right|\right)\right]
\end{aligned}
$$




$$
\begin{aligned}
\leq & \frac{L}{b}+\frac{\bar{d}}{b}-\frac{\underline{d}+A}{b} \\
& +\frac{1}{b} \min \{b(\underline{d}+B)+\underline{d}+A-\bar{d}-L, L-b(\bar{d}+A)-\bar{d}-B+\underline{d}\} \\
\leq & B_{n}
\end{aligned}
$$

and

$$
\begin{aligned}
\left(S_{L} x\right)_{n}= & \frac{L}{b}+\frac{d_{n+\tau}}{b}-\frac{x_{n+\tau}}{b}+\frac{1}{b} \sum_{i=n+\tau}^{\infty} \frac{1}{a_{i}}\left[f\left(i, x_{f_{1}(i)}, x_{f_{2}(i)}, \ldots, x_{f_{k}(i)}\right)\right. \\
& \left.-\sum_{j=i}^{\infty}\left(g\left(j, x_{g_{1}(j)}, x_{g_{2}(j)}, \ldots, x_{g_{k}(j)}\right)-c_{j}\right)\right] \\
\geq & \frac{L}{b}+\frac{d}{b}-\frac{\bar{d}+B}{b}-\frac{1}{b} \sum_{i=T}^{\infty} \frac{1}{\left|a_{i}\right|}\left[Q_{i}+\sum_{j=i}^{\infty}\left(W_{j}+\left|c_{j}\right|\right)\right] \\
\geq & \frac{L}{b}+\frac{d}{b}-\frac{\bar{d}+B}{b} \\
& -\frac{1}{b} \min \{b(\underline{d}+B)+\underline{d}+A-\bar{d}-L, L-b(\bar{d}+A)-\bar{d}-B+\underline{d}\} \\
\geq & A_{n},
\end{aligned}
$$

which imply (3.12). Consequently $S_{L}$ is a contraction mapping in $\Omega\left(\left\{A_{n}\right\}_{n \in \mathbb{Z}_{\beta}},\left\{B_{n}\right\}_{n \in \mathbb{Z}_{\beta}}\right)$ and it has a unique fixed point $x=\left\{x_{n}\right\}_{n \in \mathbb{Z}_{\beta}} \in \Omega\left(\left\{A_{n}\right\}_{n \in \mathbb{Z}_{\beta}},\left\{B_{n}\right\}_{n \in \mathbb{Z}_{\beta}}\right)$. It follows from (3.19) that

$$
\begin{aligned}
x_{n}= & \frac{L}{b}+\frac{d_{n+\tau}}{b}-\frac{x_{n+\tau}}{b}+\frac{1}{b} \sum_{i=n+\tau}^{\infty} \frac{1}{a_{i}}\left[f\left(i, x_{f_{1}(i)}, x_{f_{2}(i)}, \ldots, x_{f_{k}(i)}\right)\right. \\
& \left.-\sum_{j=i}^{\infty}\left(g\left(j, x_{g_{1}(j)}, x_{g_{2}(j)}, \ldots, x_{g_{k}(j)}\right)-c_{j}\right)\right], \quad n \geq T,
\end{aligned}
$$

which gives

$$
\begin{aligned}
\Delta\left(x_{n}+b x_{n-\tau}-d_{n}\right)= & -\frac{1}{a_{n}}\left[f\left(n, x_{f_{1}(n)}, x_{f_{2}(n)}, \ldots, x_{f_{k}(n)}\right)\right. \\
& \left.-\sum_{j=n}^{\infty}\left(g\left(j, x_{g_{1}(j)}, x_{g_{2}(j)}, \ldots, x_{g_{k}(j)}\right)-c_{j}\right)\right], \quad n \geq T+\tau
\end{aligned}
$$

and

$$
\begin{aligned}
& \Delta\left(a_{n} \Delta\left(x_{n}+b x_{n-\tau}-d_{n}\right)\right)+\Delta f\left(n, x_{f_{1}(n)}, x_{f_{2}(n)}, \ldots, x_{f_{k}(n)}\right) \\
& \quad+g\left(n, x_{g_{1}(n)}, x_{g_{2}(n)}, \ldots, x_{g_{k}(n)}\right)=c_{n}, \quad n \geq T+\tau .
\end{aligned}
$$

That is, $x=\left\{x_{n}\right\}_{n \in \mathbb{Z}_{\beta}} \in \Omega\left(\left\{A_{n}\right\}_{n \in \mathbb{Z}_{\beta}},\left\{B_{n}\right\}_{n \in \mathbb{Z}_{\beta}}\right)$ is a bounded nonoscillatory solution of equation (1.9). 
It follows from (3.11), (3.16), (3.17), and (3.19) that for any $n \geq T$ and $m \in \mathbb{N}_{0}$

$$
\begin{aligned}
\left|z_{m+1}-x_{n}\right|= & \mid\left(1-\alpha_{m}-\beta_{m}\right) z_{m, n}+\alpha_{m}\left\{\frac{L}{b}+\frac{d_{n+\tau}}{b}-\frac{z_{m, n+\tau}}{b}\right. \\
& +\frac{1}{b} \sum_{i=n+\tau}^{\infty} \frac{1}{a_{i}}\left[f\left(i, z_{m, f_{1}(i)}, z_{m, f_{2}(i)}, \ldots, z_{m, f_{k}(i)}\right)\right. \\
& \left.\left.-\sum_{j=i}^{\infty}\left(g\left(j, z_{m, g_{1}(j)}, z_{m, g_{2}(j)}, \ldots, z_{m, g_{k}(j)}\right)-c_{j}\right)\right]\right\}+\beta_{m} \gamma_{m, n}-x_{n} \mid \\
\leq & \left(1-\alpha_{m}-\beta_{m}\right)\left|z_{m, n}-x_{n}\right|+\alpha_{m}\left|\left(S_{L} z_{m}\right)_{n}-\left(S_{L} x\right)_{n}\right|+\beta_{m}\left|\gamma_{m, n}-x_{n}\right| \\
\leq & \left(1-\alpha_{m}-\beta_{m}\right)\left\|z_{m}-x\right\|+\alpha_{m} \theta\left\|z_{m}-x\right\|+2(\bar{d}+B) \beta_{m} \\
& \leq\left(1-(1-\theta) \alpha_{m}\right)\left\|z_{m}-x\right\|+2(\bar{d}+B) \beta_{m},
\end{aligned}
$$

which yields (3.5). Thus Lemma 2.1, (3.6), and (3.7) ensure that $\lim _{m \rightarrow \infty} z_{m}=x$. The rest of the proof is similar to that of Theorem 3.1 and is omitted. This completes the proof.

Theorem 3.4 Let $b \in(-\infty,-1)$, $A$ and $B$ be two positive constants with $B>A+\frac{b-2}{b+1}(\bar{d}-\underline{d})$. Assume that there exist four real sequences $\left\{P_{n}\right\}_{n \in \mathbb{N}_{n_{0}}},\left\{Q_{n}\right\}_{n \in \mathbb{N}_{n_{0}}},\left\{R_{n}\right\}_{n \in \mathbb{N}_{n_{0}}}$ and $\left\{W_{n}\right\}_{n \in \mathbb{N}_{n_{0}}}$ satisfying (3.1)-(3.3). Then

(a) for each $L \in(B+b(\underline{d}+B)+\bar{d}-\underline{d}, A+b(\bar{d}+A)+\underline{d}-\bar{d})$, there exist $\theta \in(0,1)$ and $T \geq n_{0}+\tau+|\beta|$ such that for each $z_{0}=\left\{z_{0, n}\right\}_{n \in \mathbb{Z}_{\beta}} \in \Omega\left(\left\{A_{n}\right\}_{n \in \mathbb{Z}_{\beta}},\left\{B_{n}\right\}_{n \in \mathbb{Z}_{\beta}}\right)$, the Mann iterative sequence with errors $\left\{z_{m}\right\}_{m \in \mathbb{N}_{0}}$ generated by the schemes (3.16) with $z_{m}=\left\{z_{m, n}\right\}_{n \in \mathbb{Z}_{\beta}} \in$ $\Omega\left(\left\{A_{n}\right\}_{n \in \mathbb{Z}_{\beta}},\left\{B_{n}\right\}_{n \in \mathbb{Z}_{\beta}}\right)$ for all $m \in \mathbb{N}_{0}$ converges to a bounded nonoscillatory solution $x=$ $\left\{x_{n}\right\}_{n \in \mathbb{Z}_{\beta}} \in \Omega\left(\left\{A_{n}\right\}_{n \in \mathbb{Z}_{\beta}},\left\{B_{n}\right\}_{n \in \mathbb{Z}_{\beta}}\right)$ of equation (1.9) and satisfies (3.5), where $\left\{\gamma_{m}\right\}_{m \in \mathbb{N}_{0}}$ is an arbitrary sequence in $\Omega\left(\left\{A_{n}\right\}_{n \in \mathbb{Z}_{\beta}},\left\{B_{n}\right\}_{n \in \mathbb{Z}_{\beta}}\right)$ with $\gamma_{m}=\left\{\gamma_{m, n}\right\}_{n \in \mathbb{Z}_{\beta}}$ for each $m \in \mathbb{N}_{0}$, $\left\{\alpha_{m}\right\}_{m \in \mathbb{N}_{0}}$ and $\left\{\beta_{m}\right\}_{m \in \mathbb{N}_{0}}$ are any sequences in $[0,1]$ satisfying (3.6) and (3.7);

(b) equation (1.9) possesses uncountably many bounded nonoscillatory solutions in $\Omega\left(\left\{A_{n}\right\}_{n \in \mathbb{Z}_{\beta}},\left\{B_{n}\right\}_{n \in \mathbb{Z}_{\beta}}\right)$.

Proof Let $L \in(B+b(\underline{d}+B)+\bar{d}-\underline{d}, A+b(\bar{d}+A)+\underline{d}-\bar{d})$. It follows from (3.3) and $b \in(-\infty,-1)$ that there exist $\theta \in(0,1)$ and $T \geq n_{0}+\tau+|\beta|$ satisfying

$$
\theta=-\frac{1}{b}-\frac{1}{b} \sum_{i=T}^{\infty} \frac{1}{\left|a_{i}\right|}\left(P_{i}+\sum_{j=i}^{\infty} R_{j}\right)
$$

and

$$
\begin{aligned}
& \sum_{i=T}^{\infty} \frac{1}{\left|a_{i}\right|}\left[Q_{i}+\sum_{j=i}^{\infty}\left(W_{j}+\left|c_{j}\right|\right)\right] \\
& \quad \leq \min \{A+b(\bar{d}+A)+\underline{d}-\bar{d}-L, L-B-b(\underline{d}+B)-\bar{d}+\underline{d}\} .
\end{aligned}
$$

Let the mapping $S_{L}$ be defined by (3.19). The rest of the proof is similar to that of Theorem 3.3 and is omitted. This completes the proof. 
Remark 3.1 Theorems 3.1-3.4 extend Theorem 1 in [5] under $p \neq \pm 1$. Theorems 3.1-3.4 improve Theorems 2.4-2.7 in [8], respectively. The examples in the fourth section reveal that Theorems 3.1-3.4 extend authentically the corresponding results in $[5,8]$.

\section{Applications}

In this section, we assume that $\left\{\gamma_{m}\right\}_{m \in \mathbb{N}_{0}}$ is an arbitrary sequence in $\Omega\left(\left\{A_{n}\right\}_{n \in \mathbb{Z}_{\beta}},\left\{B_{n}\right\}_{n \in \mathbb{Z}_{\beta}}\right)$ with $\gamma_{m}=\left\{\gamma_{m, n}\right\}_{n \in \mathbb{Z}_{\beta}}$ for each $m \in \mathbb{N}_{0},\left\{\alpha_{m}\right\}_{m \in \mathbb{N}_{0}}$ and $\left\{\beta_{m}\right\}_{m \in \mathbb{N}_{0}}$ are any sequences in $[0,1]$ satisfying (3.6) and (3.7).

Now we display four examples as applications of the results presented in Section 3.

Example 4.1 Consider the second order nonlinear neutral delay difference equation

$$
\begin{aligned}
& \Delta\left[(-1)^{\frac{n(n+1)}{2}} n^{3} \Delta\left(x_{n}+\frac{1}{2} x_{n-\tau}+6(-1)^{n}\right)\right]+\Delta\left(n x_{n^{2}+3 n^{2}}^{2} x_{n+(-1)^{n}}^{3}\right) \\
& \quad+\frac{x_{3 n^{4}+2 n^{2}+8 n-2}^{2}}{n^{2}\left(1+x_{n^{3}+3 n^{2}+10 n-6}^{2}\right)}=\frac{n^{2}(2 n-1)+\sqrt{3 n^{2}-2 n+1}}{n^{6}+n \ln \left(1+n^{2}\right)}, \quad n \geq 1,
\end{aligned}
$$

where $n_{0}=1$ and $\tau \in \mathbb{N}$ is fixed. Let $k=2, b=\frac{1}{2}, A=43, B=56, \alpha=0, \beta=1-\tau, \underline{d}=-6$, $\bar{d}=6$ and

$$
\begin{aligned}
& a_{n}=(-1)^{\frac{n(n+1)}{2}} n^{3}, \quad c_{n}=\frac{n^{2}(2 n-1)+\sqrt{3 n^{2}-2 n+1}}{n^{6}+n \ln \left(1+n^{2}\right)}, \quad d_{n}=6(-1)^{n}, \\
& f(n, u, v)=n u^{2} v^{3}, \quad g(n, u, v)=\frac{u^{2}}{n^{2}\left(1+v^{2}\right)}, \\
& f_{1}(n)=n^{2}+3 n, \quad f_{2}(n)=n+(-1)^{n}, \\
& g_{1}(n)=3 n^{4}+2 n^{2}+8 n-2, \quad g_{2}(n)=n^{3}+3 n^{2}+10 n-6, \\
& P_{n}=10^{12} n, \quad Q_{n}=10^{9} n, \\
& R_{n}=\frac{10^{7}}{n^{2}}, \quad W_{n}=\frac{3844}{n^{2}}, \quad(n, u, v) \in \mathbb{N}_{n_{0}} \times[\underline{d}+A, \bar{d}+B]^{2} .
\end{aligned}
$$

It is easy to show that the conditions (3.1)-(3.3) are satisfied. It follows from Theorem 3.1 that equation (4.1) possesses uncountably bounded nonoscillatory solutions in $\Omega\left(\left\{A_{n}\right\}_{n \in \mathbb{Z}_{\beta}},\left\{B_{n}\right\}_{n \in \mathbb{Z}_{\beta}}\right)$, and for any $L \in(A+b(\bar{d}+B), B+b(\underline{d}+A))$, there exist $\theta \in(0,1)$ and $T \geq n_{0}+\tau+|\beta|$ such that the Mann iterative sequence with error $\left\{z_{m}\right\}_{m \geq 0}$ generated by (3.4) converges to a bounded nonoscillatory solution $x=\left\{x_{n}\right\}_{n \in \mathbb{Z}_{\beta}} \in \Omega\left(\left\{A_{n}\right\}_{n \in \mathbb{Z}_{\beta}},\left\{B_{n}\right\}_{n \in \mathbb{Z}_{\beta}}\right)$ of equation (4.1) and (3.5) holds. Obviously, Theorem 1 in [5] and Theorem 2.5 in [8] are invalid for equation (4.1).

Example 4.2 Consider the second order nonlinear neutral delay difference equation

$$
\begin{gathered}
\Delta\left[\left(n^{2}+6\right) \Delta\left(x_{n}-\frac{1}{5} x_{n-\tau}+9 \sin \left(n^{3}+1\right)\right)\right]+\Delta\left(\frac{\sqrt{n-2} x_{2 n-3}^{3}}{n^{2}+x_{2 n^{2}-9}^{2}}\right) \\
+\frac{n^{3} x_{7 n-12}^{4}-(-1)^{n} x_{n^{2}-6}^{9}}{n^{7}+2 n^{5}+3 n^{4}+2 n+1}=\frac{\sqrt{n^{2}-n+1}}{n^{3}+3 n^{2}+4 n+1}, \quad n \geq 2,
\end{gathered}
$$


where $n_{0}=2$ and $\tau \in \mathbb{N}$ is fixed. Let $k=2, b=-\frac{1}{5}, A=11, B=23, \alpha=-5, \beta=\min \{2-\tau,-5\}$, $\underline{d}=-9, \bar{d}=9$, and

$$
\begin{aligned}
& a_{n}=n^{2}+6, \quad c_{n}=\frac{\sqrt{n^{2}-n+1}}{n^{3}+3 n^{2}+4 n+1}, \quad d_{n}=9 \sin \left(n^{3}+1\right), \\
& f(n, u, v)=\frac{\sqrt{n-2} u^{3}}{n^{2}+v^{2}}, \quad g(n, u, v)=\frac{n^{3} u^{4}-(-1)^{n} v^{9}}{n^{7}+2 n^{5}+3 n^{4}+2 n+1}, \\
& f_{1}(n)=2 n-3, \quad f_{2}(n)=2 n^{2}-9, \quad g_{1}(n)=7 n-12, \quad g_{2}(n)=n^{2}-6, \\
& P_{n}=\frac{\left(5242880+3072 n^{2}\right) \sqrt{n-2}}{n^{4}}, \quad Q_{n}=\frac{32768 \sqrt{n-2}}{n^{2}}, \\
& R_{n}=\frac{131072 n^{3}+65536 \times 10^{9}}{n^{7}}, \quad W_{n}=\frac{1048576 n^{3}+262144 \times 10^{9}}{n^{3}+1}, \\
& \quad(n, u, v) \in \mathbb{N}_{n_{0}} \times[\underline{d}+A, \bar{d}+B]^{2} .
\end{aligned}
$$

It is clear that the conditions (3.1)-(3.3) are fulfilled. It follows from Theorem 3.2 that equation (4.2) possesses uncountably bounded nonoscillatory solutions in $\Omega\left(\left\{A_{n}\right\}_{n \in \mathbb{Z}_{\beta}}\right.$, $\left.\left\{B_{n}\right\}_{n \in \mathbb{Z}_{\beta}}\right)$, and for any $L \in(A+b(\underline{d}+A), B+b(\bar{d}+B))$, there exist $\theta \in(0,1)$ and $T \geq$ $n_{0}+\tau+|\beta|$ such that the Mann iterative sequence with error $\left\{z_{m}\right\}_{m \geq 0}$ generated by (3.4) converges to a bounded nonoscillatory solution $x=\left\{x_{n}\right\}_{n \in \mathbb{Z}_{\beta}} \in \Omega\left(\left\{A_{n}\right\}_{n \in \mathbb{Z}_{\beta}},\left\{B_{n}\right\}_{n \in \mathbb{Z}_{\beta}}\right.$ ) of equation (4.2) and (3.5) holds. However, Theorem 1 in [5] and Theorem 2.6 in [8] are not applicable for equation (4.2).

Example 4.3 Consider the second order nonlinear neutral delay difference equation

$$
\begin{aligned}
& \Delta\left[n^{2}\left(1-n^{3}\right) \Delta\left(x_{n}+2 x_{n-\tau}+\frac{n(2 n-1)}{n^{2}+2}\right)\right]+\Delta\left(n^{3} x_{n^{2}+1} \sin \left(x_{n^{2}+1} x_{2 n-5}\right)\right) \\
& \quad+\frac{x_{n^{3}-2 n+1}^{5}}{n^{2}+x_{n^{2}+2 n-1}^{2}}=\frac{(-1)^{n-1} 2 n-5}{3 n^{4}+2 n^{2}+n+1}, \quad n \geq 2,
\end{aligned}
$$

where $n_{0}=2$ and $\tau \in \mathbb{N}$ is fixed. Let $k=2, b=2, A=90, B=100, \alpha=-1, \beta=\min \{2-\tau,-1\}$, $\underline{d}=\frac{1}{3}, \bar{d}=2$ and

$$
\begin{aligned}
& a_{n}=n^{2}\left(1-n^{3}\right), \quad c_{n}=\frac{(-1)^{n-1} 2 n-5}{3 n^{4}+2 n^{2}+n+1}, \quad d_{n}=\frac{n(2 n-1)}{n^{2}+2}, \\
& f(n, u, v)=n^{3} u \sin (u v), \quad g(n, u, v)=\frac{u^{5}}{n^{2}+v^{2}}, \quad f_{1}(n)=n^{2}+1, \quad f_{2}(n)=2 n-5, \\
& g_{1}(n)=n^{3}-2 n+1, \quad g_{2}(n)=n^{2}+2 n-1, \quad P_{n}=10^{5} n^{3}, \quad Q_{n}=102 n^{3}, \\
& R_{n}=\frac{10^{9} n^{2}+10^{13}}{n^{4}}, \quad W_{n}=\frac{10^{11}}{n^{2}}, \quad(n, u, v) \in \mathbb{N}_{n_{0}} \times[\underline{d}+A, \bar{d}+B]^{2} .
\end{aligned}
$$

Clearly, the conditions (3.1)-(3.3) hold. It follows from Theorem 3.3 that equation (4.3) possesses uncountably bounded nonoscillatory solutions in $\Omega\left(\left\{A_{n}\right\}_{n \in \mathbb{Z}_{\beta}},\left\{B_{n}\right\}_{n \in \mathbb{Z}_{\beta}}\right)$, and for any $L \in(B+b(\bar{d}+A)+\bar{d}-\underline{d}, A+b(\underline{d}+B)+\underline{d}-\bar{d})$, there exist $\theta \in(0,1)$ and $T \geq n_{0}+\tau+|\beta|$ such that the Mann iterative sequence with error $\left\{z_{m}\right\}_{m \geq 0}$ generated by (3.14) converges to a bounded nonoscillatory solution $x=\left\{x_{n}\right\}_{n \in \mathbb{Z}_{\beta}} \in \Omega\left(\left\{A_{n}\right\}_{n \in \mathbb{Z}_{\beta}},\left\{B_{n}\right\}_{n \in \mathbb{Z}_{\beta}}\right.$ ) of equation (4.3) 
and (3.5) holds. But Theorem 1 in [5] and Theorem 2.7 in [8] are not valid for equation (4.3).

Example 4.4 Consider the second order nonlinear neutral delay difference equation

$$
\begin{gathered}
\Delta\left[n^{2} \Delta\left(x_{n}-3 x_{n-\tau}+2(-1)^{\frac{n(n+1)(n+2)}{3}}\right)\right]+\Delta\left((-1)^{n^{2}+3 n-1} \sqrt{n-3} x_{n^{3}+n-9} x_{3 n-12}\right) \\
+\frac{x_{n^{2}+n-6}}{n^{2}+x_{2 n-5}^{2}}=\frac{(-1)^{n-1}\left(2 n^{2}-5\right)+(-1)^{n+1}(3 n+4) \ln \left(1+n^{3}\right)}{n^{4}+3 n^{3}+2 n^{2}+5 n+7}, \quad n \geq 3,
\end{gathered}
$$

where $n_{0}=3$ and $\tau \in \mathbb{N}$ is fixed. Let $k=2, b=-3, A=1, B=5, \alpha=-3, \beta=\{3-\tau,-3\}$, $\underline{d}=-2, \bar{d}=2$ and

$$
\begin{aligned}
& a_{n}=n^{2}, \quad c_{n}=\frac{(-1)^{n-1}\left(2 n^{2}-5\right)+(-1)^{n+1}(3 n+4) \ln \left(1+n^{3}\right)}{n^{4}+3 n^{3}+2 n^{2}+5 n+7}, \\
& d_{n}=2(-1)^{\frac{n(n+1)(n+2)}{3}}, \quad f(n, u, v)=(-1)^{n^{2}+3 n-1} \sqrt{n-3} u v, \\
& g(n, u, v)=\frac{u}{n^{2}+v^{2}}, \quad f_{1}(n)=n^{3}+n-9, \quad f_{2}(n)=3 n-12, \quad g_{1}(n)=2 n-5, \\
& g_{2}(n)=n^{2}+n-6, \quad P_{n}=14 \sqrt{n-3}, \quad Q_{n}=49 \sqrt{n-3}, \\
& R_{n}=\frac{14+n^{2}}{n^{4}}, \quad W_{n}=\frac{7}{n^{2}}, \quad(n, u, v) \in \mathbb{N}_{n_{0}} \times[\underline{d}+A, \bar{d}+B]^{2} .
\end{aligned}
$$

It is not difficult to verify that the conditions (3.1)-(3.3) are fulfilled. It follows from Theorem 3.4 that equation (4.4) possesses uncountably bounded nonoscillatory solutions in $\Omega\left(\left\{A_{n}\right\}_{n \in \mathbb{Z}_{\beta}},\left\{B_{n}\right\}_{n \in \mathbb{Z}_{\beta}}\right)$, and for any $L \in(B+b(\underline{d}+B)+\bar{d}-\underline{d}, A+b(\bar{d}+A)+\underline{d}-\bar{d})$, there exist $\theta \in(0,1)$ and $T \geq n_{0}+\tau+|\beta|$ such that the Mann iterative sequence with error $\left\{z_{m}\right\}_{m \geq 0}$ generated by (3.14) converges to a bounded nonoscillatory solution $x=\left\{x_{n}\right\}_{n \in \mathbb{Z}_{\beta}} \in$ $\Omega\left(\left\{A_{n}\right\}_{n \in \mathbb{Z}_{\beta}},\left\{B_{n}\right\}_{n \in \mathbb{Z}_{\beta}}\right)$ of equation (4.4) and (3.5) holds. However, Theorem 1 in [5] and Theorem 2.4 in [8] are unapplicable for equation (4.4).

\section{Competing interests}

The authors declare that they have no competing interests.

\section{Authors' contributions}

All authors read and approved the final manuscript.

\section{Author details}

'Basic Teaching Department, Dalian Vocational Technical College, Dalian, Liaoning 116035, People's Republic of China. ${ }^{2}$ Center for Studies of Marine Economy and Sustainable Development, Liaoning Normal University, Dalian, Liaoning 116029, People's Republic of China. ${ }^{3}$ Department of Mathematics and RINS, Gyeongsang National University, Jinju, 660-701, Korea.

Received: 18 August 2015 Accepted: 18 November 2015 Published online: 01 December 2015

\section{References}

1. Agarwal, RP: Difference Equations and Inequalities, 2nd edn. Dekker, New York (2000)

2. Cheng, SS, Li, HJ, Patula, WT: Bounded and zero convergent solutions of second order difference equations. J. Math. Anal. Appl. 141, 463-483 (1989)

3. González, C, Jiménez-Melado, A: Set-contractive mappings and difference equations in Banach spaces. Comput. Math. Appl. 45, 1235-1243 (2003)

4. Guo, ZM, Yu, JS: Multiplicity results for periodic solutions to second-order difference equations. J. Dyn. Differ. Equ. 18, 943-960 (2006)

5. Jinfa, C: Existence of a nonoscillatory solution of a second-order linear neutral difference equation. Appl. Math. Lett. 20, 892-899 (2007)

6. Li, X, Zhu, D: New results for the asymptotic behavior of a nonlinear second-order difference equation. Appl. Math. Lett. 16, 627-633 (2003) 
7. Liu, Z, Kang, SM, Ume, JS: Existence of uncountably many bounded nonoscillatory solutions and their iterative approximations for second order nonlinear neutral delay difference equations. Appl. Math. Comput. 213, 554-576 (2009)

8. Liu, Z, Xu, YG, Kang, SM: Global solvability for a second order nonlinear neutral delay difference equation. Comput. Math. Appl. 57, 587-595 (2009)

9. Ma, M, Guo, Z: Homoclinic orbits and subharmonics for nonlinear second order difference equations. Nonlinear Anal. 67, 1737-1745 (2007)

10. Meng, Q, Yan, J: Bounded oscillation for second-order nonlinear difference equations in critical and non-critical states. J. Comput. Appl. Math. 211, 156-172 (2008)

11. Tang, $\mathrm{XH}$ : Bounded oscillation of second-order neutral difference equations of unstable type. Comput. Math. Appl. 44, 1147-1156 (2002)

12. Thandapani, E, Arul, R, Raja, PS: Bounded oscillation of second order unstable neutral type difference equations. J. Appl. Math. Comput. 16, 79-90 (2004)

13. Thandapani, E, Arul, R, Raja, PS: The asymptotic behavior of nonoscillatory solutions of nonlinear neutral type difference equations. Math. Comput. Model. 39, 1457-1465 (2004)

14. Yu, JS, Guo, ZM, Zou, XF: Positive periodic solutions of second order self-adjoint difference equations. J. Lond. Math. Soc. 71, 146-160 (2005)

15. Liu, LS: Ishikawa and Mann iterative process with errors for nonlinear strongly accretive mappings in Banach spaces. J. Math. Anal. Appl. 194, 114-125 (1995)

\section{Submit your manuscript to a SpringerOpen ${ }^{\circ}$ journal and benefit from:}

- Convenient online submission

Rigorous peer review

- Immediate publication on acceptance

- Open access: articles freely available online

- High visibility within the field

- Retaining the copyright to your article 